\title{
Review Paper on Various Treatment Process of Pharmaceutical Wastewater
}

\author{
Mr. Umesh Shirke ${ }^{1}$, Dr. Arif Khan ${ }^{2}$ \\ ${ }^{1}$ M.Tech Student. ${ }^{2}$ Principal \\ Department of Environmental Engineering, Nuva College of Engineering \& Technology, Nagpur.
}

DOI: 10.46335/IJIES.2020.5.11.4

\begin{abstract}
Pharmaceutical compounds are typically produced in batch processes leading to the presence of a wide variety ofproducts in wastewaters which are generated in different operations, wherein copious quantities of water are used for washing of solid cake, or extraction, or washing of equipment. The wastewaters generated in different processes in the manufacture of pharmaceuticals and drugs contain a wide variety of compounds. Further, reuse of water after removal of contaminants, whether pharmaceuticals or otherwise, is required by industry. In view of the scarcity of water resources, it is necessary to understand and develop methodologies for treatment of pharmaceutical wastewater as part of water management. In this review, the various sources of wastewaters in the pharmaceutical industry are identified and the best available technologies to remove them are critically evaluated. No single technology can completely remove pharmaceuticals from wastewaters.
\end{abstract}

Keywords- Physico-chemical, Biological Process, BOD, COD, Waste Water, Suspended Solids, ASP

\section{I- INTRODUCTION}

We will restrict this review to pharmaceutical water, wherein it is widely used as a raw material, ingredient, and solvent in the processing, formulation, and manufacture of pharmaceutical products, APIs and intermediates, compendia articles, and analytical reagents.
Chemical synthesis processes use organic and inorganic chemicals in batch operations to produce drugs with defined pharmacological action or intermediates.

a series of chemical reactions are carried out in multipurpose reactors. The products are isolated by using different separation processes such as liquid-liquid extraction, leaching (solid-liquid extraction), crystallization, and filtration. The product is then usually dried, milled, and sent for further processing to the formulation unit.

The product usually in the mother liquor is transferred internally using process vessels and pipelines and thus the process becomes more complex leading to a widespread use of raw water at every step.

Wastewaters from chemical synthesis operations are diverse due to many operations and reactions taking place in the reactor as well as at different stages.

Almost every stage produces mother liquor that contains unreacted reactants, products, co products/byproducts, and residual products in the organic solvent base. Acids, bases, halides, nitrates, sulfates, cyanides, and metals may also be generated. Usually, the spent solvent recovery leads to solvent wastewater at the scrubber stage after evaporation. Wastewater is generated at the purification steps comprising solvents, finished products, cleaning water, and spills. Wastewaters from synthesis processes typically have high biological oxygen demand (BOD), chemical oxygen demand (COD), and total suspended solids (TSS) levels and $\mathrm{pH}$ ranging from 1 to 11. 


\section{II-WATER CONSUMPTION IN PHARMACEUTICAL BULK MANUFACTURING PROCESS}

A wide variety of products are made in the chemical and pharmaceutical manufacturing industries, typically requiring large volumes of chemicals, materials, and substances that are used throughout process operations. The mixtures of pharmaceuticals, hormones, and other wastewater contaminants can occur at low concentrations in streams that are susceptible to various wastewater sources, and the volumes will vary from industry to industry or site to site for the same compound. Waste streams generated in these industries can be heavily laden with contaminants, toxins,nutrients, and organics, presenting unique challenges in terms of treatment in view of stringent regulations. It is important that for reuse in both validated and nonvalidated systems the treated wastewater quality must exceed the feedwater quality for high operational efficiency, water quality, and product safety.

\subsection{Characteristics of pharmaceutical wastewater}

In general, the composition of pharmaceutical wastewater is complex, which has high concentration oforganic matter, microbial toxicity, high salt, and it's hard to biodegrade [2,3]. In addition, most of pharmaceutical factories are batch process, and there are different raw materials and production process, which causes huge varieties in different wastewater [4]. Table1 provided a summary of the characteristics of pharmaceutical wastewater [5].

Table 1-Common Pharmaceutical Wastewater Physicochemical Properties

\begin{tabular}{|c|c|c|c|c|c|c|c|}
\hline $\begin{array}{c}\text { COD } \\
(\mathrm{mg} / \\
\mathrm{L})\end{array}$ & $\begin{array}{c}\mathrm{BO} \\
\mathrm{D} 5 \\
(\mathrm{mg} / \\
\mathrm{L})\end{array}$ & $\begin{array}{c}\mathrm{TN} \\
(\mathrm{mg} \\
/ \mathrm{L})\end{array}$ & $\begin{array}{c}\mathrm{TP} \\
(\mathrm{mg} / \\
\mathrm{L})\end{array}$ & $\begin{array}{c}\mathrm{SS} \\
(\mathrm{mg} / \\
\mathrm{L})\end{array}$ & $\begin{array}{c}\text { Chromat } \\
\text { icity } \\
(\text { times })\end{array}$ & $\begin{array}{c}\text { Temp } \\
\left({ }^{\circ} \mathrm{C}\right)\end{array}$ & $\begin{array}{c}\mathrm{p} \\
\mathrm{H}\end{array}$ \\
\hline 1000 & 50 & $500-$ & $500-$ & $200-$ & $500-1000$ & $25-$ & $1-$ \\
- & $0-$ & 150 & 250 & 500 & & 80 & 8 \\
1000 & 25 & 0 & & & & & \\
0 & 00 & & & & & & \\
\hline
\end{tabular}

Otherwise, different kinds of pharmaceutical wastewater has different characteristics. Biopharmaceutical wastewater is characterized as strong fluctuation in quantity, low $\mathrm{C} / \mathrm{N}$, high $\mathrm{SS}$ concentration, high sulfate concentration, complicated composition, biological toxicity and high chroma. Chemical pharmacy is lack of nutrition, hard to biodegrade and toxicity to microbiology, and it also has high salt content.

\section{III-TREATMENT OF PHARMACEUTICAL WASTEWATER}

The pharmaceutical industry employs a wide array of wastewater treatment and disposal methods. Wastewatersgenerated from these industries vary not only in composition but also in quantity, by plant, season, and even time, depending on the raw materials and the processes used in the manufacturing of various pharmaceuticals.

However, the analysis of published information in the public domain shows that six general approaches are employed to treat pharmaceutical wastewaters which are (i) recovery of individual APIs or drugs which are likely to be present in wash waters and solvents, (ii) physical-chemical treatment by sedimentation or floatation,

(iii) aerobic/anaerobic biological treatment in membrane bioreactors or bio aeration, (iv) inactivation of activesubstances by UV oxidation in conjunction with $\mathrm{O} 3$ or $\mathrm{H} 2 \mathrm{O} 2$, (v) sterilization and decontamination of infectious andbioactive substances from biotechnology, and (vi) new hybridtechnologies specific to the pharmaceutical industry. An attempt is made here to discuss some of these issues with reference to general methodology and specific examples.

\section{IV-RECOVERY PROCESSES}

Pretreatment and recovery of various useful byproducts, such as solvents, acids, heavy metals, and various important API's, which find their way into the waste streams comprise a very important waste control strategy for pharmaceutical plants. In the fermentation plants, the fermentation broth contains large amounts of solvent and mycelia. The solvents exhibit very high BOD strength, and also some of the solvents are not biologically degradable.

Recovery of the pharmaceutical product can reduce or even eliminate waste disposal costs of the primary unit process and raw water requirements of the secondary unit process, quickly offsetting waste-treatment operational costs and improving the economics of the process. The recovered waste stream can be used elsewhere in the process, and the water could be used for boiler feed or cooling towers and other operations thereby reducing consumption of precious raw water and drastically reducing operating costs. In fact, hot waste streams after processing can be used for other heat exchangers (heat pinching) or as boiler feed thereby reducing water and energy costs. In general, pharmaceuticals have molecular weights higher than 250 Da and can be recovered by using effective membrane technologies provided that the product is alone in the 
stream .Indeed, a lot of economic benefit can be realized by using reverse osmosis, nano filtration, and ultra filtration. The filtrate can then be subjected to further processing as given in what follows.

\section{V -WASTEWATER TREATMENT OF DILUTE STREAMS}

The dilute streams from the manufacturing units are mainly treated by biological treatment methods as they convert most of the waste into gases and sludge can be disposed off harmlessly.

Available treatments include the activated sludge process, trickling filtration, the powdered-carbon-fed activated sludge process, and the anaerobic hybrid reactor.

Apart from the foregoing conventional treatment processes there are several other oxidation processes,

membrane techniques, and advanced oxidation processes.43 Based upon an extensive literature survey of the research carried out on actual pharmaceutical waste treatment, a listing has been made of the treatment technologies available in Table 2. To have a clear understanding of the various techniques used in the treatment and disposal of various types of wastes produced in the pharmaceutical industry, the treatment processes can be divided into the following four categories and subcategories:

(i) Biological treatment process:

(a) Aerobic Treatment

(b) Anaerobic Treatment

(ii) Advanced treatments:

(1) Coagulation and Sedimentation

(2) Flotation

(3) Activated Carbon Adsorption

(4) Advanced Oxidation Processes

(a) Wet air oxidation (WAO)

(b) Supercritical water oxidation (SCWO)

(c) Fenton reagent

(d) Photocatalytic oxidation

(e) Ultrasound oxidation

(f) Electrochemical oxidation

(g) Ozonation

(5) Membrane Separation

(a) Microfiltration

(b) Ultrafiltration

(c) Reverse Osmosis

(d) Electrodialysis
5.1 Biological Treatment. Biological treatment methods have been traditionally employed for dealing with pharmaceutical wastewater.44 The biological treatment of pharmaceutical wastewater includes both aerobic and anaerobic treatment systems.

Apart from the previously mentioned two processes, Afzalet al.45 investigated an efficient degradation by using Pseudomonas aeruginosa ( $\mathrm{P}$. aeruginosa) and $\mathrm{P}$. pseudomallei where the former showed a higher degradation rate and COD and BOD removal which indicated that the strains work well for phenolic wastewaters from fermentation processes.

5.1.1 Aerobic Treatment. Aerobic treatment is one of the common technologies applied which include the activated sludge process(ASP), extended aeration activated sludge process,

AS with granular activated carbon, and membrane bioreactors. $^{46-50}$

5.1.1.1 Activated Sludge Process -The activated sludge process is the most common aerobic treatment which has been found to be efficient for various categories of pharmaceutical wastewaters. ${ }^{51}$ The conventional activated sludge (CAS) treatment is a low-cost method which depends mainly on two parameters, the temperature and the hydraulic retention time (HRT). Apart from these the presence of organic matter, COD, $\mathrm{BOD}, \mathrm{pH}$, presence of non-biodegradable matter are other factors which affect the efficiency of AS method. Peng46 achieved 99\% nitrogen removal efficiency at 23 ${ }^{\circ} \mathrm{C}$. The nitrite reduction efficiency was suitable for high ammonia content wastewater and in a reduced cost. Tekin52 obtained $98 \%$ COD removal for a

Fenton pretreated manufacturing process wastewater using an aerobic sequential batch reactor. Ibuprofen, naproxen, bezafibrate, ethynilestradiol and several other estrogens show a high degree of removal efficiency but sulfa drugs like sulfomethaxazole, carbamezapine and diclofenac showed limited removal. ${ }^{43,53}$

5.1.1.2 Membrane Bioreactors. : In the past decade, use of membrane bioreactors (MBRs) for pharmaceutical wastewater treatment has gained much attention as it is a technically and economically feasible alternative for water and wastewater treatment, especially because of high sludge retention time (SRT) achieved within compact reactor volumes. In the MBR the concentration of microorganisms can be increased to up to $20 \mathrm{mg} / \mathrm{L} .54$ This high concentration of biomass increases the degradation capacity of larger organic molecules. Another advantage of membrane treatment is separation of suspended solids by membranes, so they are not limited by the settling characteristics of the sludge. 55 Removal efficiencies of $98.7 \%$ for TSS and $90.4 \%$ for 
total COD were achieved for a MBR coupled with conventional activated sludge reactor in a study carried out54 for wastewaters comprised of analgesics and antiinflammatory drugs (ibuprofen, diclofenac, indomethacin, and acetaminophen), antibiotics (ofloxacin, sulfamethoxazole, and erythromycin), and $\beta$ blockers (atenolol and metoprolol).54 Noble56 used a hollow fiber submerged MBR, for fermentation process wastewater having a very high COD of around 40000 $\mathrm{mg} / \mathrm{L}$. More than $90 \% \mathrm{COD}$ and $98 \%$ BOD removal was achieved.

In the case of membrane processes, a general approach to produce clean water from dirty or polluted water in the pharmaceutical industry will be based on the size of the pollutants in the following order: bacteria particles (microfiltration), macromolecules and viruses (ultrafiltration), divalent ions (Nano filtration), and monovalent ions (reverse osmosis).

\subsubsection{Anaerobic Treatment -}

Anaerobic treatment has been done by using continuous stirred tank reactors (anaerobic digestion), fluidized bed reactors, and up-flow anaerobic sludgereactors, etc. ${ }^{63-68}$ Anaerobic hybrid reactors, which are a combination of suspended growth and attached growth systems, have recently gained much attention. The significance of anaerobic treatment over aerobic processes is the ability to deal with high concentration wastewater, with lesser energy inputs, low sludge yield, low operation cost, and economical byproduct recovery of biomethane as a valuable energy source.43 Up-flow anaerobic batch reactor (USAR) has been shown to be very efficient in removal of high concentrations of $\mathrm{PhP}$ 's from pharmaceutical wastewater. ${ }^{65,69}$ A USAR operating at higher temperatures of about $55{ }^{\circ} \mathrm{C}$ showed a high COD (65-75\%) and BOD (80-94\%) removal even at a very high concentration of organic content of $9 \mathrm{~kg}$ of $\mathrm{COD} /(\mathrm{m} 3 \cdot$ day).70 It is shown that $75 \%$ COD removal and more than $95 \%$ tylosin removal from an antibiotic effluent wastewater is possible thereby making USAR a suitable application for such wastewaters. In a study by Kang et al.,71 catalytic wet air oxidation was employed with anaerobic biological oxidation to high COD (70000-120000 $\mathrm{mg} / \mathrm{L}$ ) containing vitamin process wastewater. With the combination, more than $94.66 \%$ COD removal was obtained with total biodegradability of the organic content. More than $60-65 \%$ removal was achieved for chemical synthesis wastewater having a COD of about $40000-60000 \mathrm{mg} / \mathrm{L}$ by using a hybrid upflow anaerobic sludge blank reactor. Specific methanogenic analysis showed no inhibitory action, and the biomass obtained was highly economical. ${ }^{66}$

\subsection{Methods of Advanced Treatment of Pharmaceutical Wastewater}

\subsubsection{Coagulation and sedimentation}

5.2.1.1 Coagulation is adding chemical agents to wastewater, dispersing by rapid mixing, then making stable pollutants into unstable and precipitable matters. The mechanism of coagulating is complex. For advanced treatment of pharmaceutical wastewater, the key is how to squeeze and remove bound water round hydrophilic colloid. So the character of flocculent is important, which related to the effect of coagulation. Inorganic metal salts and polymers are frequently used as flocculent. This method can remove SS, chromaticity and toxic organic matter [8]. Meanwhile, it can improve the biodegradability of pharmaceutical wastewater.

5.2.1.2 Sedimentation is the most common method after coagulation. Under the gravity, pollutants can be separated, which has greater density than wastewater.

Coagulation and sedimentation have some advantages, such as easy operation and mature

technology, but it is hard to remove dissolved organic matter.

\subsubsection{Flotation}

Except for sedimentation, flotation can also remove suspended solids of secondary effluent. The

technology characteristic is producing a large number of tiny bubbles by injecting air into wastewater, forming floating floc with smaller density than wastewater. And it can float to the surface of wastewater to separate.

\subsubsection{Activated carbon adsorption}

Activated carbon, as a kind of adsorbent, has many advantages. It has large specific surface area, multilevel pore structure, high adsorption capacity and stable chemical property. Therefore, it is widely used as adsorbent or catalyst carrier to remove pollutants $[9,10]$. In industrial effluents treatment, activated carbon is used for effluent, which is toxic and hard to achieve discharge standard.It is an important method of advanced treatment of pharmaceutical wastewater as well.

Activated carbon adsorption can be classified as physical adsorption and chemical adsorption.

Physical adsorption is reversible, and no selectivity to adsorbate. When activated carbon saturated by adsorbates, it is easy to desorb. To the contrary, chemical adsorption adsorbs only one or several specific adsorbates, which is irreversible and hard to desorption. For cyclic utilization, saturation of activated carbon restores its adsorption property by regeneration.

This method is widely used for advanced treatment, because it can be recycled, its better treatment effect and 
wide suitability. But there are some disadvantages, such as high costs relatively, low efficiency of regeneration and complex operation, which limit application.

\subsubsection{Advanced oxidation processes}

Advanced oxidation processes (AOPs), which can oxidize pollutants by forming free radicals. Those kinds of pollutants cannot be degraded by common oxidizing agent. There are many kinds of AOPs, such as wet air oxidation, supercritical water oxidation, Fenton reagent, photocatalytic oxidation, ultrasound oxidation, electrochemical oxidation and ozonation.

5.2.4.1. Wet air oxidation(WAO)- WAO has been put forward by F. J. Zimmer Mann in 1958, which was used for papermaking black liquid treatment. By using of air or oxygen as the oxidant, this method decomposes organic matter into inorganic or small molecules at high temperature $\left(150-350{ }^{\circ} \mathrm{C}\right)$ and high pressure (0.5-20 $\mathrm{Mpa})$. WAO is generally used in pretreatment of wastewater advanced treatment.

This method has wide range of applications, high efficiency of COD removal, which can even reaches more than $90 \%$ under appropriate conditions, low energy consumption, less secondary pollution, and it is easy management.

\subsubsection{Supercritical water oxidation(SCWO)- SCWO} is chemical reaction between dissolved oxygen and organic pollutants in supercritical water. Organic matter, air, and supercritical water were complete mixed at 24 Mpa pressure and $400{ }^{\circ} \mathrm{C}$ temperature, becoming homogeneous phase. Under these conditions, organic compounds spontaneously initiate the oxidation reaction. With the increase of the reaction temperature, $99.9 \%$ or more of the organic matter is rapidly oxidized into simple non-toxic small molecules in a period of time, achieving the purpose of removing pollutants. SCWO has high oxidation efficiency, will not cause secondary pollution, organic can be oxidized completely. However, this method has some shortcomings, such as it requires high operating conditions and high cost.

5.2.4. 3. Fenton reagent - Fenton system was initially used in organic synthesis, with the deepening of people's understanding of it, the system was gradually applied to industrial wastewater treatment. Fenton reaction can be carried out under normal temperature and pressure, and less damage to the environment [11]. It is an advanced oxidation method, which is simple, mild reaction conditions, andhigh oxidation efficiency. However, the shortcomings of the Fenton reaction cannot be ignored.
One is due to free radical scavenging and decomposition of $\mathrm{H} 2 \mathrm{O} 2$ itself, leading the loss of oxidant. The other is the formation of iron mud in the neutral conditions, making it hard to the subsequent processing[12].

\subsubsection{Photocatalytic oxidation-Photochemical} oxidation, also known as ultraviolet photocatalyticoxidation, is a method combination of UV radiation and oxidant. In the excitation of ultraviolet light, oxidative decomposition of oxidants produces stronger oxidative capacity of free radicals, which can oxidize more difficult to decomposition of refractory organic pollutants with oxidants only. According to the types of oxidants, photochemical oxidation can be divided into UV / O3, UV / H2O, UV / H2O2 / O3 and so on. At present, the steps of the reaction process still can't be described. However, the effect of this method on the treatment of refractory organic matter is undeniable.

5.2.4.5. Ultrasound oxidation-Ultrasonic degradation of organic matter in wastewater is a physical and chemical degradation process, mainly based on ultrasonic cavitation effect and the resulting physical and chemical changes, which includes the following three ways: free radical oxidation, pyrolysis and supercritical water oxidation. There are many factors that affect the effect of ultrasonic degradation of organic pollutants in wastewater. The main factors are ultrasound frequency, sound power, ultrasonic time, solution temperature and $\mathrm{pH}$, and the nature of organic matter.

5.2.4.6. Electrochemical oxidation - The use of electrochemical reaction to remove toxic and harmful pollutants in the water, is known as the electrochemical method. Electrochemistry is a new method in water treatment field. The principle of the method is as follow: in the electrochemical reaction process, the reactant will lost electrons and be oxidized in the anode. Conversely, the reactant in the cathode will lose electrons and be reduced. In general, the removal of refractory organic matter, mainly due to the oxidation of the anode. Conventional electrochemical wastewater treatment processes includes: electrolytic recovery, electrochemical oxidation, electrolytic air flotation, electrodialysis and micro-electrolysis.

Electrochemical method is also known as "Environmentally Friendly" process, has a great advantage compared with other methods. For example, the electrochemical method is generally carried out under normal temperature and pressure and has high efficiency, can be used alone or in combination with other processes, and it covers a small area and no 
secondary pollution, has relatively high degree of automation. The main research direction of the electrochemical reaction in the future is the research of the anode and electrochemical reactor [13].

5.2.4.7. Ozonation- For a long time, ozone is considered to be a very effective oxidant and disinfectant. In acidic conditions, ozone is primarily an oxidant. But in neutral and alkaline conditions, it mainly relys on free radical reactions. Table 2 shows the oxidation potential of various oxidants. It can be seen that the oxidation capacity of ozone is significantly higher than other conventional oxidants.

Ozone can quickly oxidize and decompose most of the organic matter in the water, which can effectively remove pollutants in water. However, it is difficult to directly mineralize. Instead, a product which is easily biodegradable can be produced. At the same time, it can also effectively remove turbidity and pathogens in wastewater. Ozone can be combined with other wastewater treatment technologies, forming ozone advanced oxidation technology. This method has stronger oxidation ability and lower selectivity to reactants, Such as O3 / H2O2, O3 / UV, etc.

\subsubsection{Membrane Separation}

Under certain driving force across the membrane, a component in water selectively permeates it by using permselective membrane separating media, which is called membrane separation. In this way,achieve the separation, purification, concentration of the target substance from the mixture. There are several membrane separation techniques in wastewater treatment. Such as microfiltration, ultrafiltration, reverse osmosis and electrodialysis.

5.2.5.1 Microfiltration(MF) - MF is based on static pressure as the driving force, and the separation process is performed by the action of the sieve separation of the membrane, which principle is similar to traditional filtration. The slight difference is that the pore size of MF is smaller. This method can effectively remove SS and microorganisms in wastewater.

\subsubsection{Ultrafiltration(UF) -Bezawadaet al.01UF} driving force is the pressure difference between the membranes on both sides, the filter medium is the ultrafiltration membrane. Under certain pressure, when water passes through the membrane surface, water, inorganic salts and small molecules penetrate, other macromolecules are trapped.

This method is mainly used for the removal of macromolecules and colloids in wastewater. In the application of this method, it should be ensured that the membrane has adequate membrane flux and is easily disassembled, replaced, cleaned.

5.2.5.3. Reverse osmosis(RO) - There are two categories of RO membrane, cellulose ester and aromatic polyamide. Its component form includes tube, plate and frame, roll and hollow fiber type. RO process can remove a wide range of impurities, dissolved inorganic salts and a variety of organic matter. Meanwhile, it has a high efficiency of salt removal and water reuse rate. However, this method requires a high pretreatment of the feed water. With the development of RO, nanofiltration(NF) has been proposed as a new method.

5.2.5.4. Electrodialysis(ED)- ED is a combination of electrolytic and dialysis diffusion process. Under the action of the DC electric field, anions and cations of the dissolved salts in the wastewater are moved to the anode and the cathode respectively. In this way, the concentration of anions and cationsin the intermediate compartment is gradually reduced, and the separation and recovery are achieved.

This method has many advantages, such as less energy and pharmaceutical consumption, less Environmental pollution, easy to operate and automate. But it can only remove the salt in water, and desalination efficiency is lower than RO.

\section{VI- CONCLUSION}

Due to the complexity of pharmaceutical processes, pharmaceutical wastewater has some characteristics, such as poor biodegradability and high concentration. From these characteristics, treatment of pharmaceutical wastewater is very necessary. There are many kinds of advanced treatment, each method has its own features. Above treatment process can effectively improve the quality of pharmaceutical wastewater effluent.

\section{REFERENCES}

[1] Recovery of Bacillus licheniformis alkaline protease from supernatant of fermented wastewater sludge using ultrafiltration and its characterization. Biotechnol.Res. Int. 2011, 1-11. Bezawada, J.; Yan, S.; John, R. P.; Tyagi, R. D.; Surampalli, R. Y. Recovery of Bacillus licheniformis alkaline protease from supernatant of fermented wastewater sludge using ultrafiltration and its characterization.Biotechnol.Res. Int. 2011, 1-11. 
International Journal of Innovations in Engineering and Science, Vol 5, No.11, 2020

\section{$w w w . i j i e s . n e t$}

[2] ChandrakanthGadipelly, $\uparrow \quad$ AntíaPérez-González,ł

Ganapati D. Yadav, ${ }^{*},+$
InmaculadaOrtiz, + RaquelIbáñez, $\neq \quad$ Virendra $K$. Rathod, $†$ and Kumudini V. Marathe Pharmaceutical Industry Wastewater: Review of the Technologies for Water Treatment and Reuse. Pubs.acs.org/IECR.

[3] Sun, M.; Gan, S. X.; Yin, D. F.; Liu, H. Y.; Yang, W. D.Application of nano-filtration membrane in the purification process of Tylosin. Chin. J. Antibiot. 2000, $25,172-174$.

[4] Wenxin M, Weizhong C, Jianjun $R$, Zhangqian $W$ and Yueming L 2001 J. Environ.Pollution.Control. 2 87-9

[5] Xin L and Guoyi L 2015 A Review: Pharmaceutical Wastewater Treatment Technology and Research in China (Zhuhai: Asia-Pacific Energy Equipment Engineering Research Conference) pp 345-7

[6] Yu Y 2013 Experimental on Pharmaceutical Tail Water before Biochemical Pretreatment (Changchun: Jilin University) pp 12-9

[7] $Y$ Guo, P S Qi and Y Z Liu State Key Laboratory of Urban Water Resource and Environment, Harbin Institute ofTechnology, Heilongjiang, China

[8] Yiping $G$ and $Y u$ B 2010 Advanced Treatment and Recycling Technology of Wastewater Treatment Plant (China Architecture Press) pp 198-206 -

[9] Bingyu M, Yubin T, Fangyan C and Tao W $2015 \mathrm{~J}$. Chinese. J. Environ. Eng. 4 1863-4

[10] Sandoval R, Cooper A M and Aymar K 2011 J. J. Hazard. Mater. 20 296-303

[11] Joseph J, Pignatello, Esther $O$ and Allison M $2006 \mathrm{~J}$. Crit. Rev. Env. Sci. Tec. 1-84

[12] Bokare A D and Choi W. 2012 J. J. Hazard. Mater. 275 $122-4$

[13] Hui W and JianLong W. 1999 J. Chin. J. Envir. Sci. 19 441-4 